\title{
Complete Response to Sorafenib in Locally Recurrent Unresectable Aggressive Fibromatosis
}

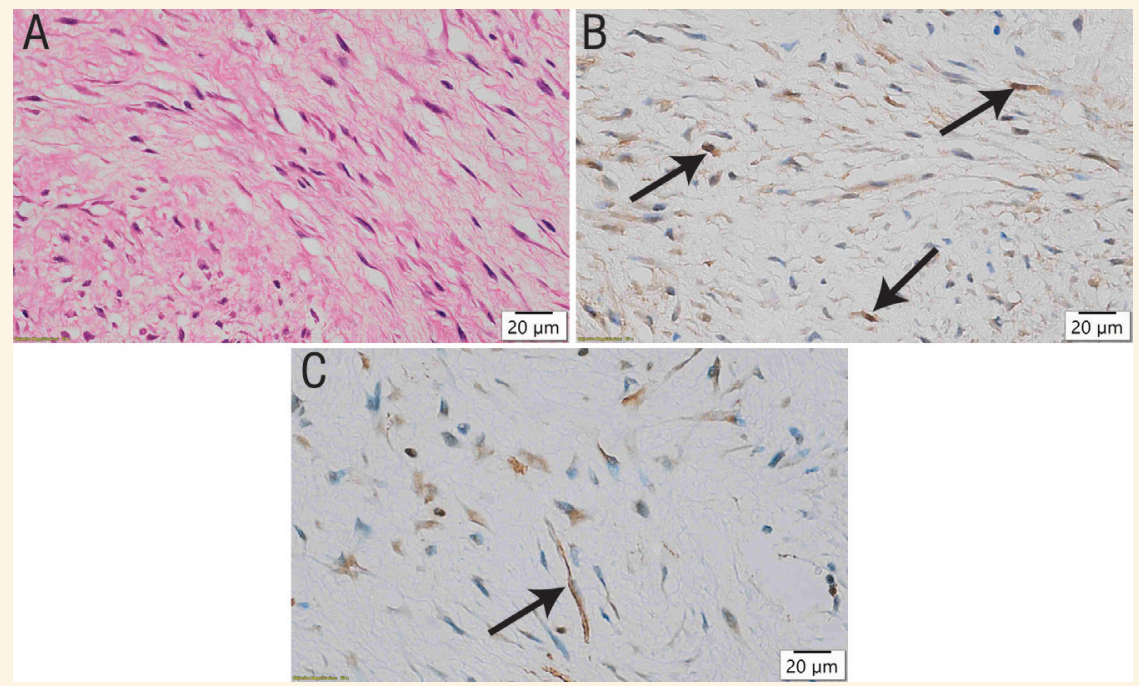

Figure 1: (A) Hematoxylin \& Eosin (H\&E) stained section of the supra-clavicular mass in a 48-year-old female patient at x200 magnification showing a bland spindle cell neoplasm consisting of sweeping intersecting fascicles, set in variable loose-to-dense fibrocollagenous stroma; (B) Beta-Catenin immunohistochemical stain at x600 magnification showing positive nuclear staining in tumour cells (arrows); (C) Smooth muscle actin immunohistochemical stain at $\mathrm{x} 400$ magnification positive in tumour cells (arrow).

A

48-YEAR-OLD FEMALE PATIENT PRESENTED with a $5 \mathrm{~cm}$ mass in the left supra-clavicular fossa of 12-months duration, progressively increasing in size. A biopsy revealed a spindle cell neoplasm arranged in long fascicles, positive for B-catenin and focally positive for smooth muscle actin [Figure 1]. A diagnosis of aggressive fibromatosis was established. The mass was surgically excised, the resection margin was positive and the diagnosis was consistent with aggressive fibromatosis. The mass recurred within three months of resection and the patient presented to the clinic with a $7 \times 5 \mathrm{~cm}$ lobulated, hyper-attenuated soft tissue mass at the left posterior cervical region extending to the infraclavicular region abutting the muscles. There were no co-morbidities and the performance status on the WHO scale was 1 . The patient was commenced on celecoxib. An MRI demonstrated a locally advanced disease [Figures 2A, B and C]. Due to the increasing pain and a lack of clinical response over three months while on celecoxib, the patient was commenced on a multi-tyrosine kinase inhibitor, sorafenib, at a standard dose of $400 \mathrm{mg}$ twice daily. Within two weeks after starting sorafenib, the patient developed a generalised rash with itching, aches and pains, fatigue, blisters over the sole of the left foot and diarrhea. The patient had then stopped using sorafenib. Full blood count, renal, liver and thyroid function tests remained within the normal limits. Three weeks later, after the rash and the blister had settled, the patient has commenced again on sorafenib at a reduced dose of $200 \mathrm{mg}$ twice daily. The patient developed grade III hand-foot syndrome as the mass clinically decreased in size. The frequency of sorafenib was reduced to once daily and the patient maintained this treatment for a total of 11 months. A follow-up MRI revealed a complete radiological response [Figures 2D, E and F]. She was then referred for adjuvant radiotherapy. 


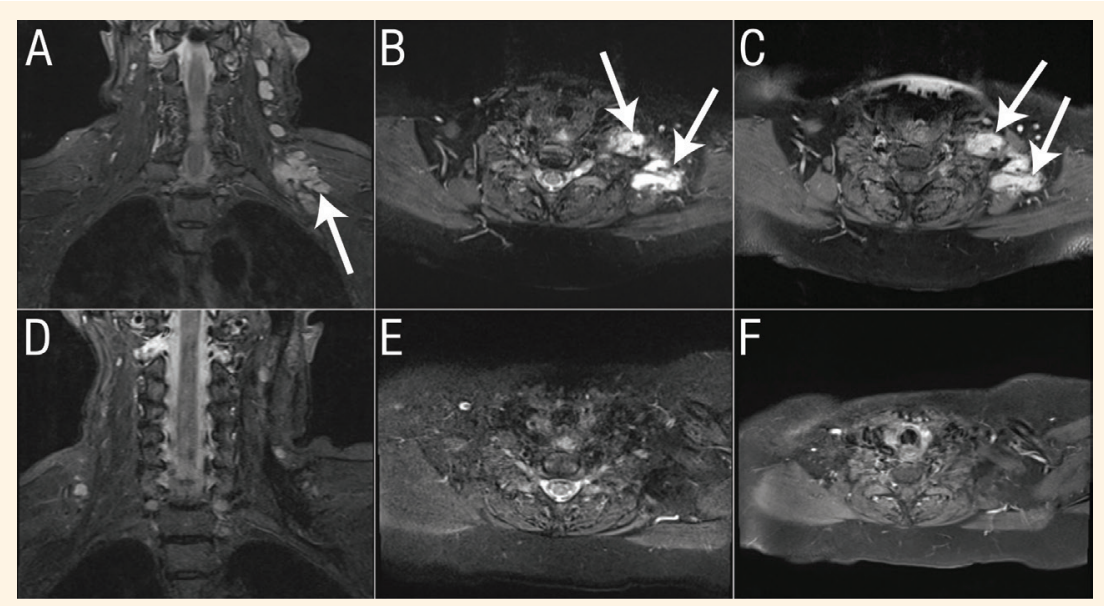

Figure 2: (A) Coronal short-TI inversion recovery (STIR) images, (B) axial STIR image at C7-T1 level and (C) axial fatsaturated contrast-enhanced T1 weighted image at C7-T1 level of a 48-year-old female patient before commencing sorafenib treatment showing a T2 hyperintense enhancing lesion in the left posterior supra-clavicular and the suprascapular regions and an ipsilateral cervical lymph node (marked with arrows). (D) Coronal STIR image, (E) axial STIR image at C7-T1 level and (F) axial fat-saturated contrast-enhanced T1 weighted image at C7-T1 level 11 months after treatment with sorafenib showing no residual disease.

\section{Comment}

Aggressive fibromatosis, also known as desmoid tumour, is a rare, locally aggressive neoplasm commonly arising from the abdominal wall's connective tissue, mesentery, and the neurovascular bundle of extremities. The vast majority of cases are sporadic and $5-16 \%$ are associated with germline mutations in the adenomatous polyposis coli (APC) gene. ${ }^{1}$ Details of presenting features, etiopathogenesis, molecular cytogenetics and treatment have been recently reviewed., ${ }^{1,2}$ Complete surgical excision remains the standard treatment, yet local recurrence risk is high and is reported to be up to $40 \%$. If excised incompletely, the tumours are known to recur rapidly. Several agents have been shown to have modest activity. ${ }^{1}$ Due to the tumour's rarity, the data are derived from a case series and retrospective analysis. $^{1,2}$ Recently, a prospective randomised trial compared sorafenib to a placebo. ${ }^{3}$ Out of the 49 patients, the objective response rate (ORR) was 33\% with only one complete response in the sorafenib group, compared to an ORR of $20 \%$ in the placebo group. The median time to objective response was 9.6 and 13.3 months in the sorafenib and the placebo groups, respectively. The 2-year progression-free survival was $81 \%$ and $36 \%$, respectively (hazard ratio $0.13 ; 0.05-0.31$ ). We report a complete response to an abbreviated sorafenib dose in a patient with locally advanced, unresectable aggressive fibromatosis.

\section{Reference}

1. Ganeshan DM, Amini B, Nikolaidis P, Assing M, Vikram R. Current update on desmoids fibromatosis. J Computer Assist Tomogr 2019; 43:29-38. https://doi.org/10.1097/RCT.00000 00000000790 .

2. Garcia-Ortega DY, Martin-Tellez KS, Cuellar-Hubbe M, Martínez-Said H, Álvarez-Cano A, Brener-Chaoul M, et al. Desmoid-type firbromatosis. Cancers (Basel) 2020; 12:1851. https://doi.org/10.3390/cancers12071851.

3. Gounder MM, Mahoney MR, Van Tine BA, Ravi V, Attia S, Deshpande HA, et al. Sorafenib for advanced and refractory desmoids tumors. N Eng J Med 2018; 379:2417-28. https://doi. org/10.1056/NEJMoa1805052. 